\title{
Energy Management Optimization of Generators Using Modified Firefly Algorithm
}

\author{
Wafeeqa Abdulrazak Hasan ${ }^{1}$, Issa Ahmed Abed ${ }^{2}$, Diyah Kammel Shary ${ }^{3}$ \\ \{Wafeeqa.alibraheem@stu.edu.iq ${ }^{1}$, issaahmedabed@stu.edu.iq ${ }^{2}$, diyahpower@stu.edu.iq $\left.{ }^{3}\right\}$
}

Basrah Engineering Technical College, Southern Technical University, Iraq ${ }^{1,2,3}$

\begin{abstract}
The proposed microgrid system in island mode in this study is made up of a mix of conventional and renewable energy sources, including two diesel generators, two wind turbines, and three fuel cell plants. Using the artificial intelligence technology to manage its energy sources by optimization methods, to decide the scheduling of each generator's optimum power output per hour over 24 hours at a minimum total generation cost using MATLAB software package. For management of the energy of the proposed microgrid in an hour-to-day, such data should be specified in advance, including the planned load demand, the wind forecast for the next day for each hour, and the cost coefficients of each generator. To investigate the optimum performance, each algorithm will be executed for 30 runs at each hour within 24 hours. The methods of optimization used in this microgrid system suggest modified of the firefly algorithm (IFA) by hybridizing it with the local search algorithm and then using IFA in this study to manage the energy sources. Eventually, the results obtained that the IFA method enhances the performance of the energy scheduling with minimise the total generation cost of microgrid compared to the other optimization methods presented in this study, which IFA method produced better results compared to the used FA.
\end{abstract}

Keywords: Energy management; optimization; microgrid firefly algorithm; modified firefly algorithm.

\section{Introduction}

To optimise the use of Distributed Generators (DGs), the power system is being reconfigured to allow for higher penetration levels. Microgrids (MG) are small power systems made up of a number of dispersed micro-generators that can meet a considerable amount of a community's demand. MGs have a number of advantages for the system, including lower customer interruption costs, lower system losses, and better renewable resource penetration levels [1]. A microgrid is one of the electrical systems that supply electrical energy gained from renewable energy sources [2]. The best solution to the energy crisis is the MG group with renewable energy sources. [3]. Microgrids are classified according to the type of operation as grid-connected mode and an islanded mode where the distributed energy resources (DERs) are supplied the loads directly without being connected to the grid [4,5]. MGs energy management aims to increase energy efficiency and minimize losses of power. An optimal arrangement of units in the grid-connected type or stand-alone type MGs energy management is carried out to optimize benefits through the application of renewable DGs also the energy storage system (ESS) [6,7]. Management of energy for the microgrid is the control software that allows the system to operate optimally [8]. The use of artificial intelligence technology to tackle problems 
with electricity source problems that use various sources due to their robustness and capability was active to handle easy use with complicated systems [3]. For these systems, the energy management system (EMS) would collect all the necessary information, solve an optimization problem, and communicate the correct allocation of energy into each distributed energy resource [9]. The proposed target concerns identifying the optimal hourly management for the ability to operate the proposed microgrid system to reach the required load demand profile at a minimum total cost of generation. Energy management strategies have always been known to be an important issue of research. Firstly, the approaches that have been suggested in the literature for optimal cost and scheduling power generation areas Sulaiman, et al. [10]. had presented an economic load dispatch issue paper that used a meta-heuristic firefly algorithm. K. Thenmalar and A. Allirani [11] presented a firefly algorithm that minimizes the total operating costs of the generator, the total emission costs as well as the scheduling of the generator units. M.N. Abdullah et.al. [12]. optimized the operation scheduling of microgrid by proposed a firefly algorithm (FA) which is used to find out the best energy production, provided the cost is as low as possible. A. Karthikeyan et.al [13] had given particle swarm optimization method (PSO) to solve the proposed economic dispatch problem which concerning the cost and emission are analysed for the 24-hour interval. F. Shariatzadeh et.al [14] proposed a solution to the problem of the shipboard microgrid electricity system (SMPS) for implemented particle optimization (PSO) approach and that the use of genetic algorithm (GA). M. Basu and A. Chowdhury [15] had been given a cuckoo search algorithm for solving both convex and no convex economic dispatch (ED) problems of fossil fuel-fired generators

This study used the optimization methods in the island microgrid system include the firefly algorithm (FA) which is an algorithm based on the original firefly algorithm and suggested modified of it (IFA) by hybridizing it with the local search algorithm to manage the energy sources in this system, also used to determine each generator's optimum power output within 24 hours at a minimal cost. Organizing the paper includes problem Formulation and microgrid system model in Section 2, Section 3 demonstrations the proposed optimization methods IFA, Next Section 4 Simulation performance and discussion. In the end, the conclusion was presented in Section 5.

\section{Microgrid System Model}

The main problem of the research is to determine the optimal scheduling of power generations in an hour - day with minimizing the total generation cost of microgrid systems while satisfying the constraints of load demand and limits generators. When a microgrid's generation consists of several sources with varying costs for each source, whether renewable or conventional, the difficulty becomes even greater. Although the running cost is the most important factor, the microgrid's performance must also be optimal. As a result, the firefly algorithm and a modified firefly method will be used to reduce the MG system's overall generating cost in island mode. The main model of a microgrid system is implemented, which includes two diesel generators, three wind turbines, and fuel-cell plants, as well as general operational and typical DERs' associated cost functions. The established plant is operating in an island microgrid system $[12,15]$. And this microgrid system is shown in Fig. 1. 


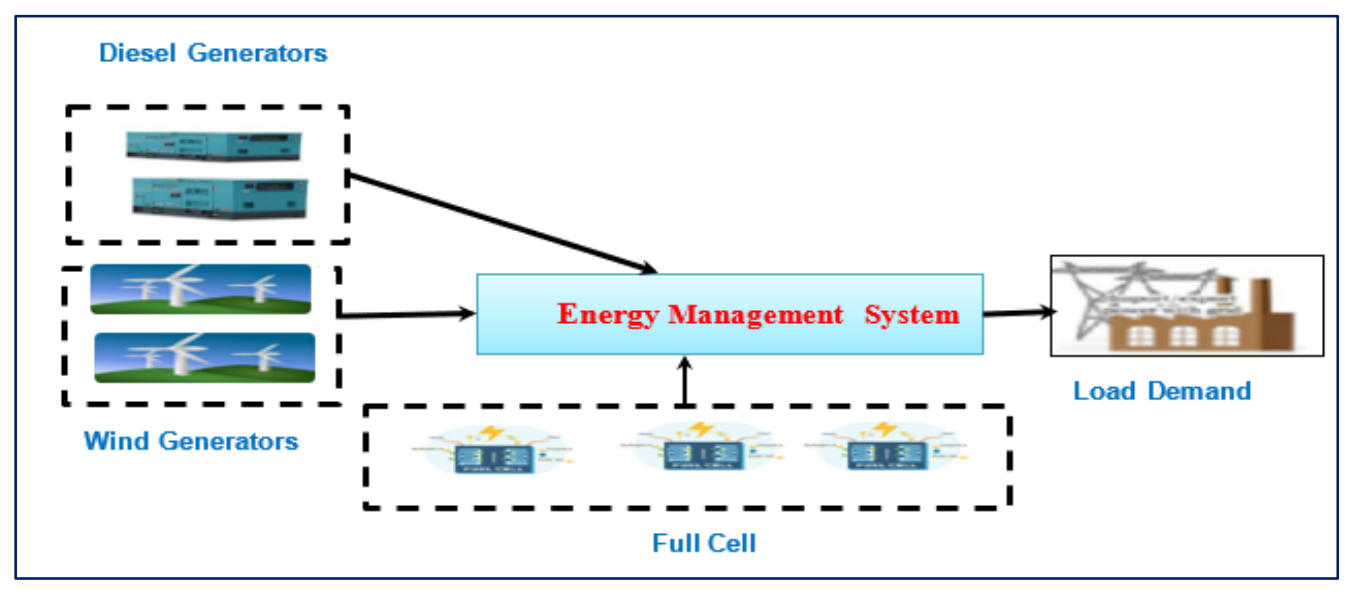

Fig. 1. The Model of a microgrid system.

\subsection{Problem Formulation}

The objective function represents the minimization of the total generation cost (FCtotal) of the microgrid, which is illustrated as follows: To evaluate the optimum power output of any generator in each hour to find the lowest cost within 24 hours of the considered microgrid, the objective function represents the minimization of the total generation cost (FCtotal):

$F C_{\text {total }}=\sum_{t=1}^{T}\left(\sum_{i=1}^{N_{\text {diesel }}} F_{D, i}(t)+\sum_{i=1}^{N_{\text {wind }}} F_{W, i}(t)+\sum_{i=1}^{N_{\text {fuel }}} F_{F, i}(t)\right)$

Where $\mathrm{N}_{\text {diesel }}, \mathrm{N}_{\text {wind, }}$ and $\mathrm{N}_{\text {fuel }}$ are the numbers of diesel generators, wind turbines, and fuel cell plants. Parameter $\mathrm{T}$ is the scheduling time, and $\mathrm{FC}_{\text {total }}$ is the overall value of cost generation throughout time T. Two different types of restrictions depending on the issue of microgrid optimisation. The first restriction is power produced by all the distributed generations should also be balanced by the power load for each scheduled time $(\mathrm{t})$, and the second restriction is each generator's output power should be within both the lower limit and the upper limit.

\section{Modified Firefly Algorithm (IFA)}

For firefly algorithms, the search depends entirely on random walks, so it is not possible to guarantee a fast convergence [16]. Local search is implemented to facilitate the algorithm's exploration and search abilities and ensure fast convergence of algorithms. Firefly algorithm has been allowed to search for a global optimum for the suggested target function [17]. Can use a local search technique to enhancement several of the target functions of features found in the population [18]. Within this development, programming codes are presents to solve the problem of optimization using IFA. Then it was implemented on the proposed MG's objective/fitness feature. In this study, the implement improved firefly algorithm. Here is a significant improvement, essentially, in the combination of a local search method with FA to speed up convergence. This makes the technique more applicable for a broader variety of practical energy management applications while retaining the attractive characteristics of its classical FA. 


\subsection{Local Search}

The principle behind the local search is to replace the current candidate solution s with some adjacent candidate solutions within the S-search area of the complete candidate solution. Candidate solution N(s) neighborhood $s \in S$ is described by all candidate solutions which can be achieved through the application of specific solutions modifies to s, or "movements"[19]. The widest perturbing form of local search is a possibly iterative improvement (In the literature often referred to as iterated descent, hill climbing, etc.). Replaces the existing candidate solution with an improved neighbour solution, as well as repeats its steps till a locally optimal candidate solution, namely a candidate, is found a solution without neighbours improving [19]. To minimize FA's shortages and increase the FA's search capability, firefly has been improved and enhanced [20].

This technique requires no gradient knowledge to conduct the local operation search. We used this technique since we wanted to demonstrate that even with a simplistic one, the algorithmic approach provides better convergence properties [18]. The pseudo-code of the local search algorithm is as follows [18].

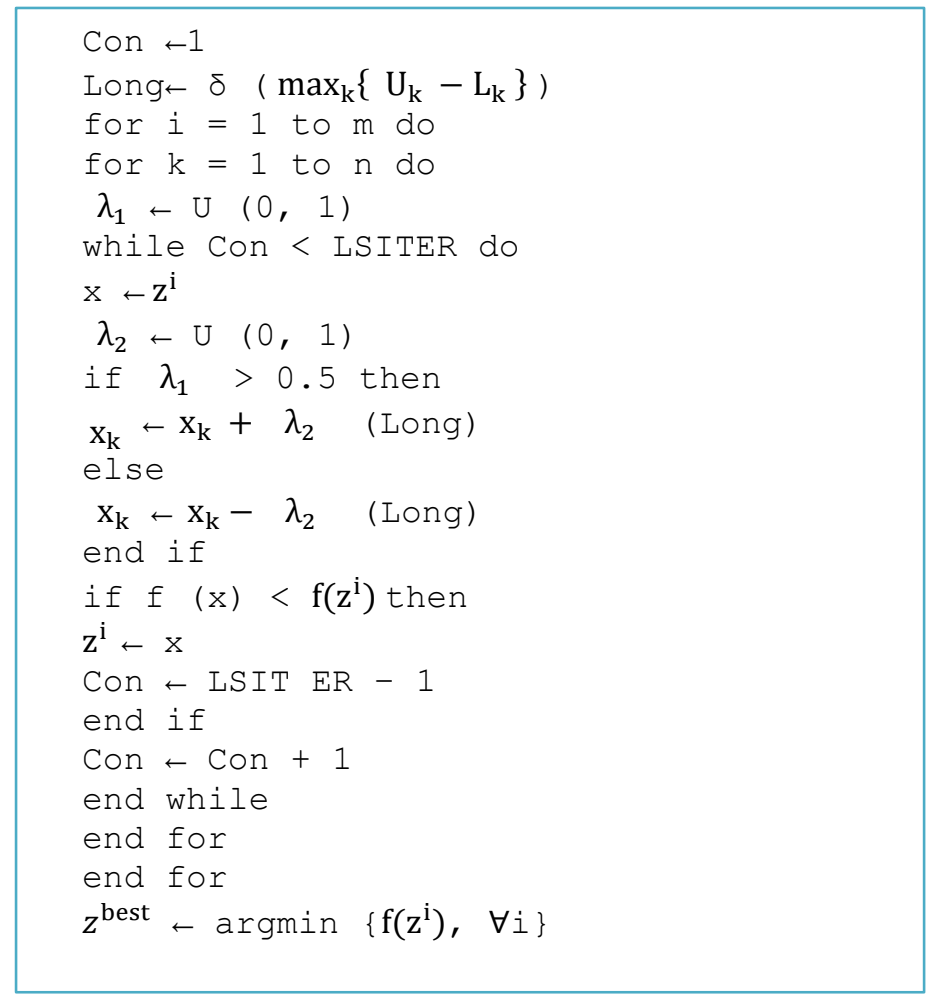

\section{Simulation Results and Discussion}

In this study for energy management issue of MG which is scheduling of optimum output power generators by running each method in (30 runs) per hour within 24 hours with the 
minimum total generation cost for each generator, which must also satisfy the load demand and each generator must produce within its limits as shown in Fig. 2.

The microgrid in this study is made up of numerous traditional and renewable energy power plants, including two diesel generators, two wind generators, and three fuel cell plants.

\subsection{Effect the Change of Population Sizes by Using IFA}

When FA test is done (30 runs) at each hour within 24 hours to achieve the minimal cost, the findings of IFA are given a lower cost of the microgrid system at a population size of 100 compared to population sizes of (20) and (50). The cost for population size (100) is $\$ 30919.9526$ dollars, which is the lowest cost when compared to other population sizes. In addition, Fig. 3 shows the distribution for the best power schedule in 24 hours. To further elucidate, Fig. 3 shows the convergence curve for various population sizes. Using IFA, Fig. 4 depicted the largest population size at the lowest cost.

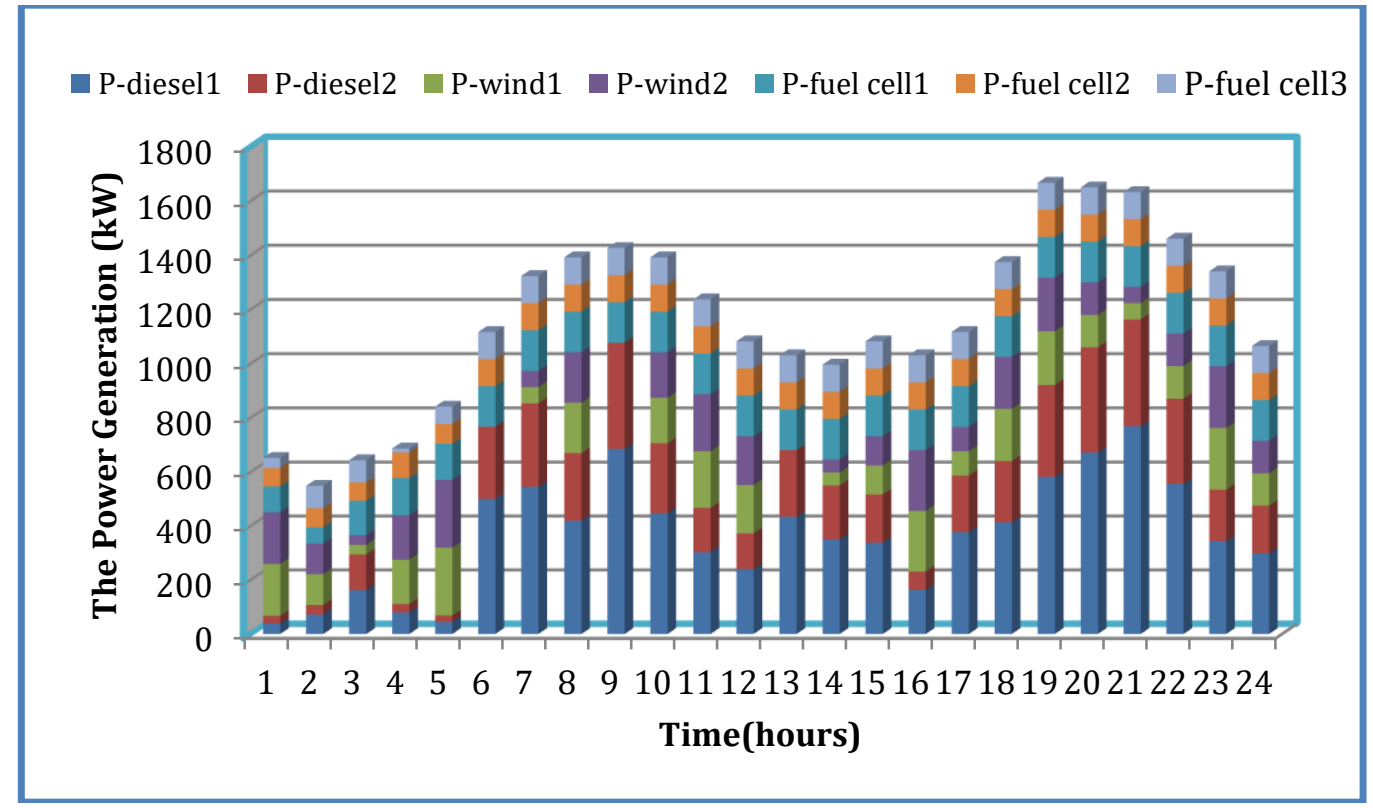

Fig. 2. Best plan of power generation of microgrid system by using IFA at (MaxIter 500) and (100 population size). 


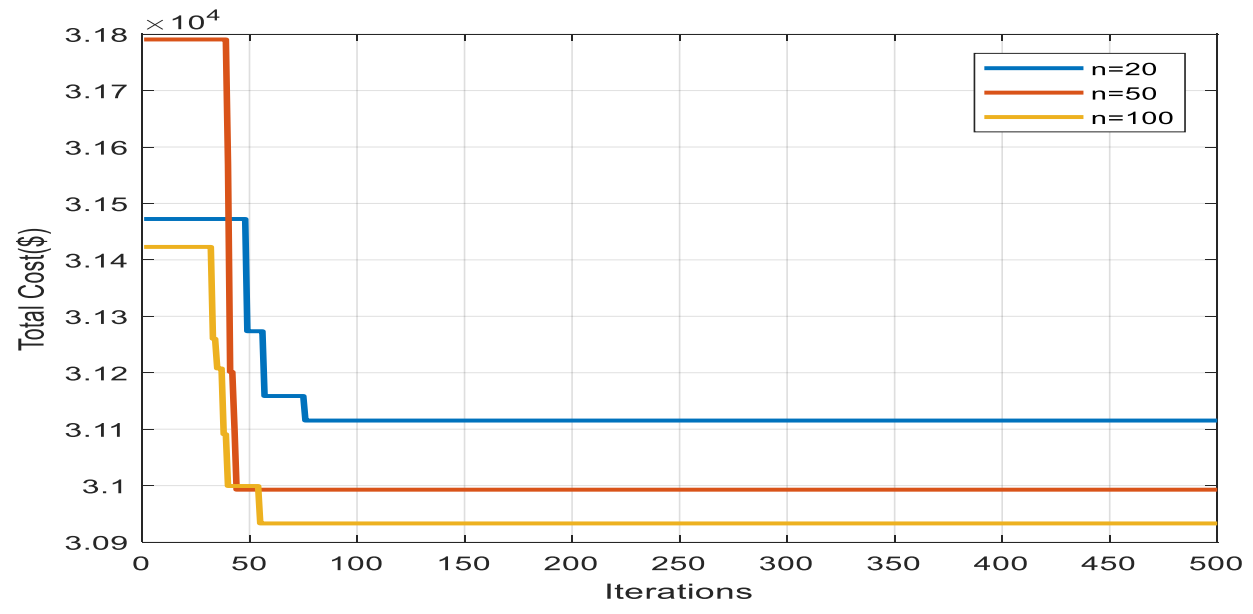

Fig. 3. Convergence curve for a number of population sizes using IFA for minimum cost.

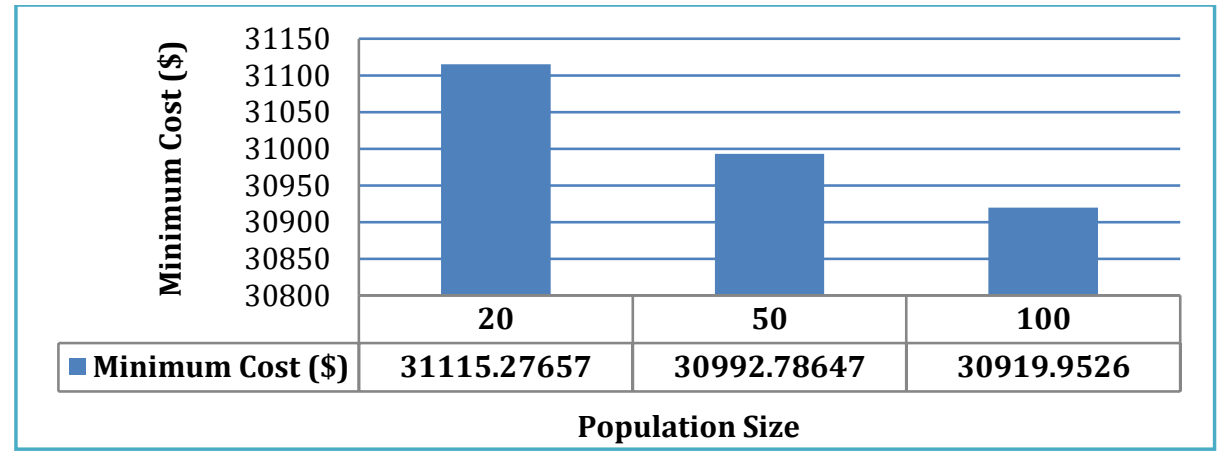

Fig. 4. Various population sizes and the lowest cost of using IFA (MaxIter 500)

\subsection{Effect Change the Number of Iteration (MaxIter) by Using IFA}

The optimization results show that the modified firefly algorithm is more effective at (100 population size) and high-value iteration than at other low-value iterations. At iteration (500), IFA performs the best operation for MG with the lowest generation cost compared to other cases (100) and (250) at system hourly load demand, as shown in Fig. 5. Fig. 6 shows the comparison of the generation power of the microgrid with that of the requested power in every hour of a day, which the results obtained from IFA. The power balance equation is seen to have been fulfilled because the power generations are in correspondence with the power demanded. There is a difference between the values of the power generation and the values of power demand for each hour because it is a very small value, so it is neglected. Fig. 7 displays the best cost at every hour after the IFA has been implemented for 30 attempts during each hour, and the sum of the best cost at each hour represents the best cost of generation within a day 


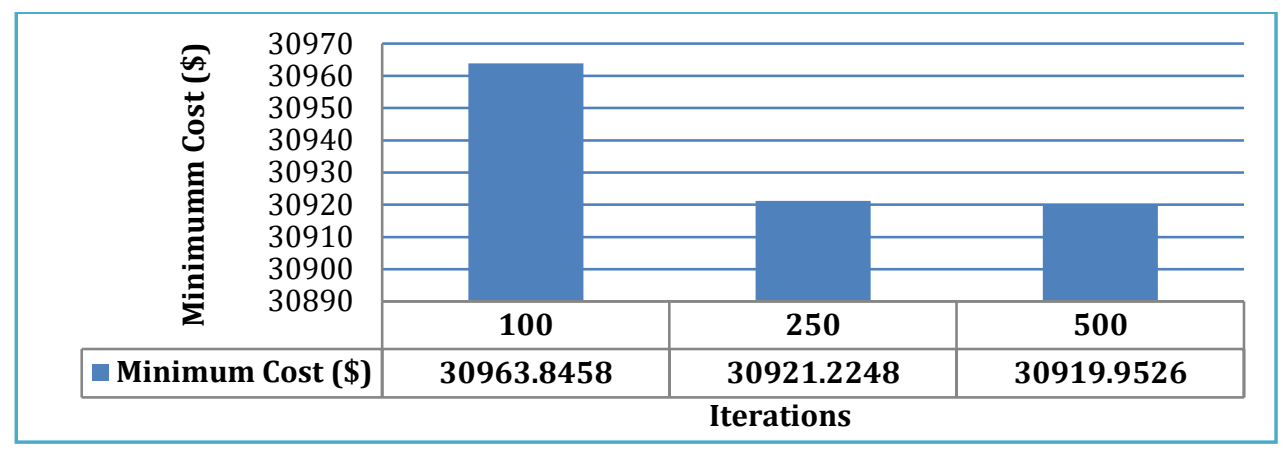

Fig. 5. Different Iteration and the minimum cost from using IFA at (population size100).

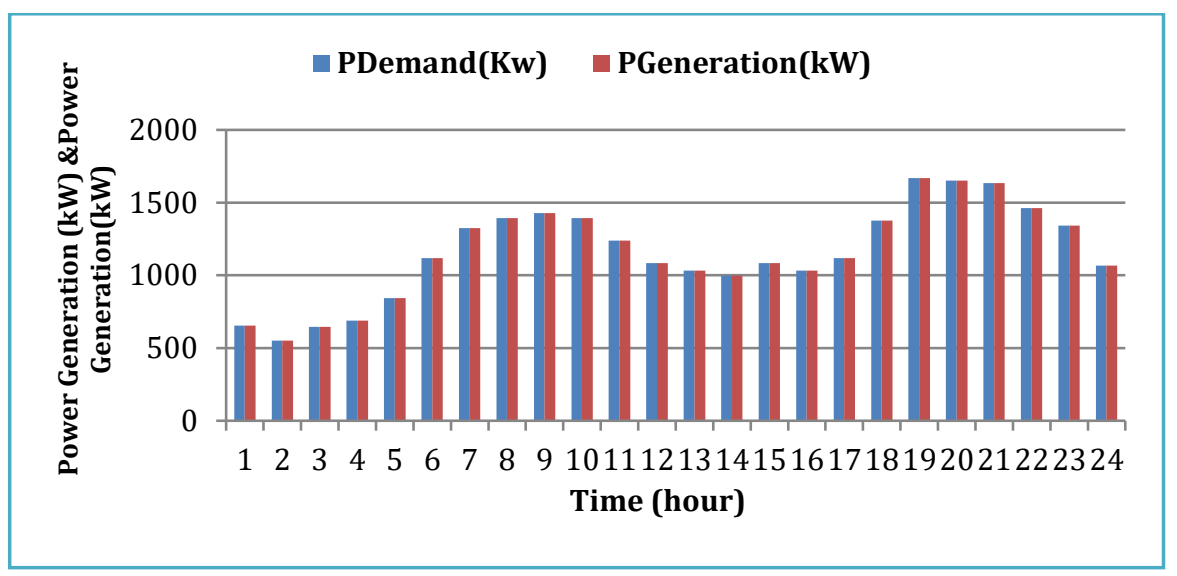

Fig. 6. Different Iteration and the minimum cost from using IFA at (population size100).

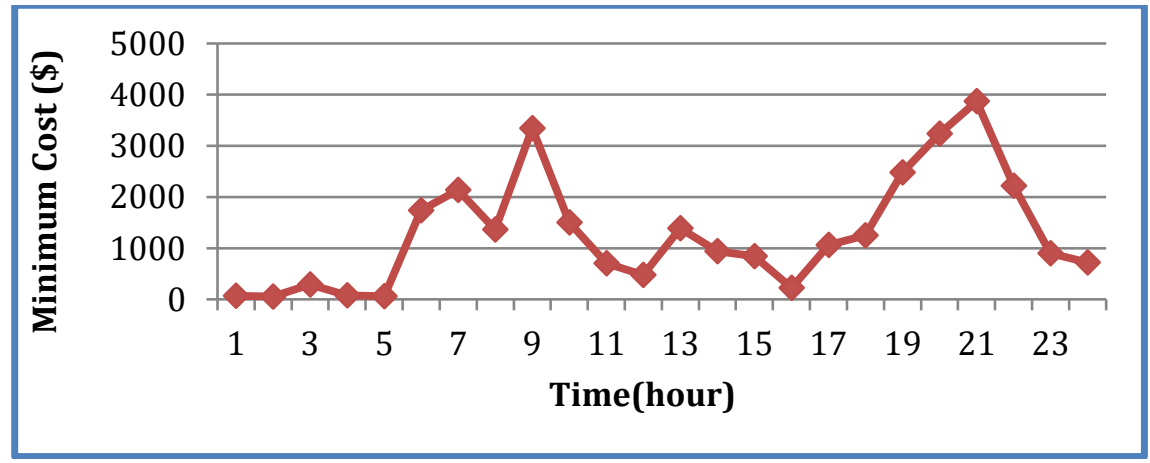

Fig. 7. Optimal hourly generation cost of the microgrid system by using IFA.

And then analyse the findings and compare them with various optimization approaches. In two cases as follows: 


\section{Case1: Change the Standard Parameters of the Algorithms}

\section{A Comparison of the Optimal Cost with Number of Iteration}

The comparative results of optimal total generation cost for the proposed microgrid system by firefly algorithm and its modified algorithm that used at the different number of iterations are (500) and (100) with some populations is (100) and each algorithm is executed in (30 times) in each hour within 24 hours and the result as shown Table (1). In this comparison notice that at maximum iteration gives the less minimum cost by used IFA than the comparison of the results with FA. When increasing the number of iterations for the IFA the total generation cost of the microgrid system is decreased.

Table 1. Comparative results of optimal generation cost by the proposed optimization methods with several iterations at (population size100).

\begin{tabular}{lll}
\hline Iteration & IFA & FA \\
\hline 500 & 30919.9526 & 30968.1056 \\
100 & 30963.8458 & 31021.754 \\
\hline
\end{tabular}

\section{Comparison of the Optimal Cost with Population Size}

The results of optimal total generation cost at several iterations are (500) with a different number of populations is (20) and (100) at each algorithm are executed at 30 runs in each hour within 24 hours at (500 iterations) and the result as shown Table (2). In this comparison notice that at maximum iteration give the less minimum cost. When increasing the population size for the various algorithms the total generation cost of the microgrid system is decreased.

Table 2. Comparative results of optimal generation cost by various methods with population size at(500 iterations).

\begin{tabular}{lll}
\hline Population size & IFA & FA \\
\hline 100 & 30919.9526 & 30968.1056 \\
20 & 31115.27657 & 31064.4187 \\
\hline
\end{tabular}

\section{Case2: Change the Number of Times of the algorithms Running.}

Original FA and its modified are executed in (3 times) for each hour within 24 hours on the MG system at same parameters of iteration is (500) and several populations are (100), where the results of minimum total generation of cost listed in Table 3 to compare it with the results in running the original FA and IFA algorithms at (30 times). From the results obtained at the number of runs of the optimization algorithm in (30 times) are less than the results when runs the optimization algorithms in (3 times). So, when increase the number runs for the various algorithms the total generation cost of the microgrid system is decreases. Figs 8 and 9, give the speed convergences of the original FA and its modified optimization method in this test at (3 runs). From the comparison between the simulation results at a different number of executed runs, notice that the optimal results are found to increase the number of runs the modified FA. 
Table 3. Comparative results of optimal generation cost by the proposed optimization methods with some iterations at (population size100).

Table 3. Comparative results of optimum generation output by various methods at (500 iterations and 100 population size).

\begin{tabular}{lll}
\hline Algorithms & IFA & FA \\
\hline Run(30)times & 30919.9526 & 30968.1056 \\
Run(3)times & 31919.7555 & 31921.6915 \\
\hline
\end{tabular}

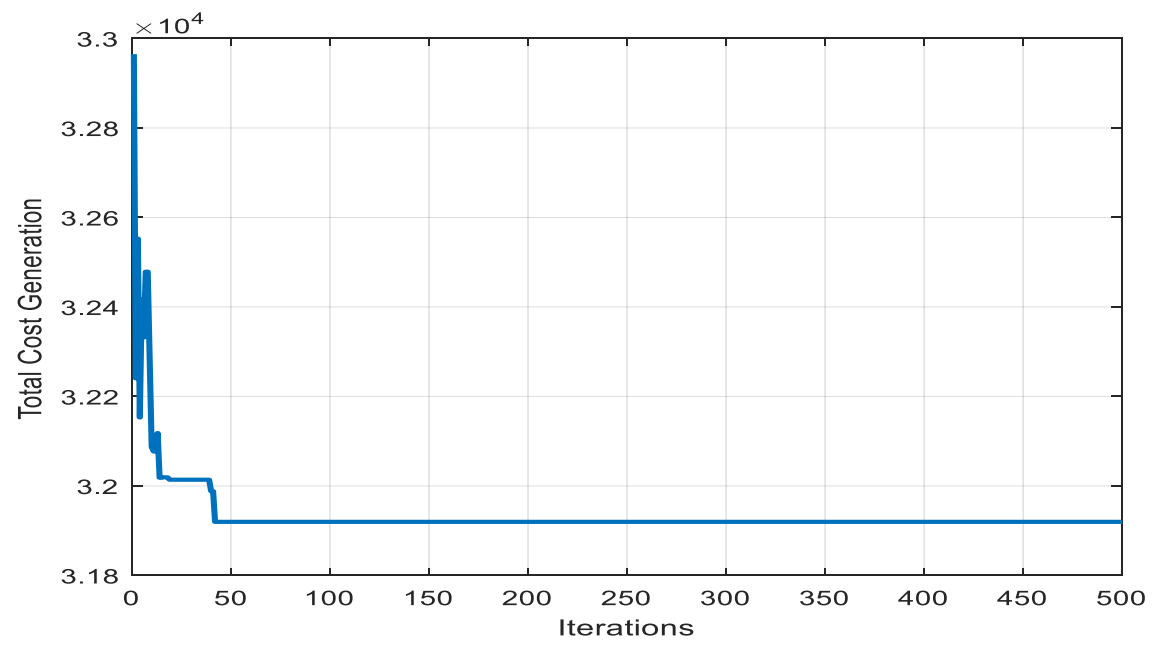

Fig. 8. Converges curve of used IFA method.

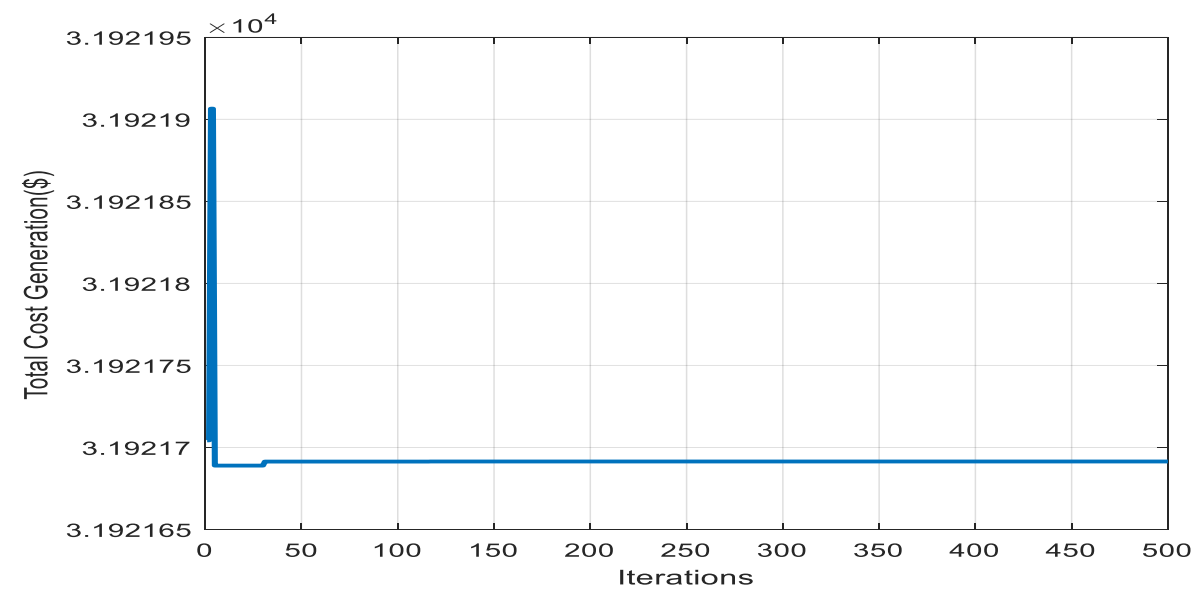

Fig. 9. Converges curve of used FA method. 


\section{Conclusion}

The optimal cost of total generation in each hour within 24 hours and scheduling optimal power generation to lower total cost of generation by applying optimization methods such as firefly algorithm, improved firefly algorithm by hybrid FA with local search to enhanced FA are discussed in this study. Add for that, presents a new method is atom search algorithm and particle swarm optimization method. Where runs each method (30 times) for each hour through day and simulation the results of each proposed method at various values of iteration and population size, which find that when increase these standard parameters of the algorithm will find best results. The light absorption coefficient, attraction coefficient minimum value, and absorption coefficient of the Firefly algorithm are all dependent on the optimised problem, and we discovered that there are two methods for selecting the appropriate algorithm parameters for the problem: one is to tune the parameters, and the other is to modify the parameters while the algorithm is running. It can be observed from the results obtained that the IFA method enhances the performance of the energy scheduling by minimizing the total generation cost of microgrid compared to the other optimization methods presented in this study, which IFA method produced better results compared to the used FA. Additionally, it can also be concluded that if the number of operating times increases for the algorithm used in this study for each hour within 24 hours, an optimum power schedule can be found at a minimum of the overall cost of generation of the microgrid system.

\section{Acknowledgments.}

We are grateful to Southern Technical University, Engineering Technical College of Basra, and the Department of Electrical Power Engineering for their contributions to this paper.

\section{References}

[1] Ashoke K. B., Sunetra. C., \&S. P. C.Impact of Strategic Deployment of CHP-Based DERs on Microgrid Reliability. Power Delivery, IEEE Transactions; 2010;25(3):1697-1705.

[2] Zhixin M, Ling X, V.Disfani, \&L. Fan, An SOC-Based Battery Management System for Microgrids. IEEE Transactions on Smart Grid;2014;5(2): 966-973.

[3] Sharmistha S.,Subhadeep B., \&Aniruddha B.Operation cost minimization of a Micro-Grid using Quasi-Oppositional Swine Influenza Model Based Optimization with Quarantine. Ain Shams Engineering Journal .2018; 9(1):45-63.

[4] Zein Alabedin, A. M., El-Saadany, E. F., \& Salama, M. M. A. Generation scheduling in Microgrids under uncertainties in power generation. IEEE Electrical Power and Energy Conference; 2012; p.133-138.

[5] Emiliano D.A., Hao Z., \& Georgios. B. G. Distributed Optimal Power Flow for Smart Microgrids .IEEE Transactions on Smart Grid;2013;4(3):1464-1475.

[6] Hossam A.G., \& Abdelazeem A.A. Microgrid energy management in grid-connected and islanding modes based on SVC. Energy Conversion and Management. 2014 :86;964-972.

[7] Ashoke K. B., S.P. C, S. Chowdhury, and S. Paul. Microgrids: Energy management by strategic deployment of DERs-A comprehensive survey. Renewable and Sustainable Energy Reviews; 2011:15(9); 4348-4356.

[8] Wencong S. \& Jianhui W. Energy Management Systems in Microgrid Operations. The Electricity Journal. 2012: 5(8);45-60.

[9] Alireza A. A Memory-Based Genetic Algorithm for Optimization of Power Generation in a Microgrid. IEEE Transactions on Sustainable Energy;2018: 9(3); 1081-1089. 
[10] Sulaiman, M. H., Mustafa, M. W., Zakaria, Z. N., Aliman, O., \& Abdul Rahim, S. R. Firefly Algorithm Technique for Solving Economic Dispatch Problemll, IEEE International Power Engineering and optimization conference, Malaysia; June 2012: 6-7; 90-95.

[11] Thenmalar, K., \& Allirani, A. Solution of firefly algorithm for the economic themal power dispatch with emission constraint in various generation plants\|, Fourth International Conference on Computing, Communications and Networking Technologies (ICCCNT); 2013.

[12] Mohd N. A. ,Nur.L.A. ,\&Jasru J.J. Opyimal Power Generation in Microgrid System using Firefly Algorithm,IEEE 20176 th International Conference on Electrical Engeenering and Informatics(ICEEI);Langkawi;Malysia;2017.

[13] Karthikeyan, A., Manikandan, K., \& Somasundaram, P.,Economic Dispatch of Microgrid with Smart Energy Storage Systems using Particle Swarm Optimization, in 2016 International Conference on Computation of Power, Energy Information and Commuincation (ICCPEIC), p. 783-789.

[14] Shariatzadeh, F., Kumar, N., \& Srivastava, A. K. Optimal Control Algorithms for Reconfiguration of Shipboard Microgrid Distribution System Using Intelligent Techniquesl, IEEE Transactions on Industry Applications; 2017 :53(1), 474-482.

[15] Mousumi. B. ,\&Anirban C. Cucko Search algorithm for economic dispatch ,Energy;2013:60;99108.

[16] Gai-Ge W.,Lihong G.,Hong D. ,\& Heqi W. A New Improved Firefly Algorithm for Global Numerical Optemization,Journal of Computational and Theortical Nanoscience;2014:11(2);477-485.

[17] Abhishek R. \&T. M. Optimal reactive power dispatch using hybrid Nelder-Mead simplex-based firefly algorithm: International Journal of Electrical Power \& Energy Systems;2015: 66 ; 9-24.

[18] Issa A. A. Photovoltaic Parameters Identification Using Optimization Algorithm. University of Thi-Qar Journal. 2017;12(2):47-64.

[19] Kwiecien J. \& B.F. Firefly algorithm in optimization of queueing systems:Bulletin of The Polish Academy of Sciences Technical Sciences; $2012: 60$ (2).

[20] Issa A. A. An Improved Technique Based on Firefly Algorithm to Estimate the Parameters of the Photovoltaic Model, Iraq, Journal Electronic Engineering ;2016:12(2);137-145. 